\title{
Le faïençage thermique à grand nombre de cycles, un phénomène lié à la structure
}

\author{
SAID TAHERI ${ }^{\mathrm{a}}$ \\ LaMSID UMR EDF-CNRS 2832, 1 Ave. Général de Gaulle, 92141 Clamart Cedex, France
}

Reçu le 25 février 2005, accepté le 21 mars 2005

\begin{abstract}
Résumé - On explique le fäiençage thermique à grand nombre de cycles d'une structure, par l'arrêt de propagation dans l'épaisseur des fissures initiées à la surface. Sur certains composants de centrales nucléaires les configurations des réseaux de faïençage près des soudures ont été reliées aux signes des contraintes résiduelles de soudage. On montre par ailleurs que les contraintes résiduelles locales de soudage dépendent de la dérivée seconde spatiale du champ de température de soudage. Loin des soudures dans les zones de fortes compressions on explique la présence du réseau par l'effet pénalisant, pour un acier austénitique, du pré-écrouissage sur la fatigue en déformation contrôlée. On conclut finalement que l'opération du grenaillage peut être pénalisante pour les aciers austénitiques en fatigue thermique.
\end{abstract}

Mots clés : Faïençage / soudage / pré-écrouissage / grenaillage / acier inoxydable

Abstract - High cycle thermal crazing, a phenomena related to the structure. High cycle thermal fatigue cracking is explained through the arrest of cracks initiated at surface, in the thickness of the component. On some components of nuclear power plants the configuration of crack network is explained through the sign of weld residual stress. We show also that local residual stresses are dependent on second derivative of temperature field. Far from the weld we explain the presence of crack network under high compressive stress for stainless steels by detrimental effect of pre-hardening on fatigue life in strain control and we conclude that shot peening may be detrimental in thermal fatigue.

Key words: Crazing / welding / pre-hardening / shot peening / stainless steel

\section{Introduction}

Le faïençage thermique à grand nombre de cycles est un problème générique des circuits de refroidissement à l'arrêt des centrales nucléaires. Il se traduit par un réseau de fissures denses, peu profondes, unidirectionnelles ou multidirectionnelles. Le travail présenté ici comporte deux volets :

- Comprendre l'existence d'un réseau dense et multidirectionnel peu profond en fatigue thermique à grand nombre de cycles quand il n'en existe pas pour les composants des réacteurs à eau pressurisée en fatigue mécanique. Ce phénomène est expliqué [1] par l'arrêt de la propagation dans l'épaisseur lié aux hautes fréquences du chargement thermique au travers du gradient de contrainte dans l'épaisseur.

- Comprendre pourquoi dans certaines zones il y a un faïençage et dans d'autres zones il y a l'absence de faïençage. On montre que la présence du fä̈ençage

a Auteur correspondant : said.taheri@edf.fr est accentuée d'une part par la contrainte moyenne de traction [2] issue du soudage et d'autre part par l'effet du pré-écrouissage, ce dernier étant pénalisant pour des aciers austénitiques en déformation contrôlée (fatigue thermique à grand nombre de cycles) en opposition avec une situation en contrainte contrôlée [3]. On montre par ailleurs que pour un tube mince les contraintes résiduelles locales de soudage dépendent de la dérivée seconde du champ de température de soudage, laquelle est très difficile à approcher à cause des aléas du soudage. Ceci peut créer une marge d'erreur difficilement compressible sur l'évaluation de contrainte locale du soudage.

\section{Explication du faïençage thermique au travers de la structure}

Dans cette partie on explique la densification et la multi-directionnalité d'un réseau de faïençage thermique. 


\section{Nomenclature}

\begin{tabular}{|ll|}
\hline$a$ & la profondeur maximale d'une fissure semi-elliptique \\
$a / c$ & l'excentricité d'une fissure semi-elliptique \\
FIC & le facteur d'intensité des contraintes \\
$h$ & l'épaisseur d'un tube \\
$K_{\mathrm{I}}(\mathrm{A})$ & le facteur d'intensité des contraintes en mode I au point A \\
$K_{\mathrm{th}}$ & le seuil de non propagation des fissures en mode I \\
$N$ & le nombre de cycles à la fatigue \\
$R_{\mathrm{m}}$ & le rayon moyen d'un tube \\
$\mathrm{MPa}$ & Méga Pascal \\
$S_{\mathrm{m}}$ & la contrainte moyenne \\
$\Delta K$ & la variation du facteur d'intensité des contraintes \\
$\Delta \sigma / 2$ & l'amplitude de contrainte \\
$\Delta \varepsilon / 2$ & l'amplitude de déformation \\
$\Delta \varepsilon_{\mathrm{p}} / 2$ & l'amplitude de déformation plastique \\
\hline
\end{tabular}

\subsection{La densification d'un réseau de fissures de faïençage}

En fatigue mécanique à grand nombre de cycles sur une éprouvette l'initiation constitue l'essentiel de la durée de vie. Quand une fissure est amorcée dans une zone de concentration de contraintes la rupture est rapidement atteinte avant que l'amorçage ne se fasse sur d'autres sites (bande de dispersion large en fatigue à grand nombre de cycles). Il y a donc une probabilité très faible de détecter un réseau de fissures. Dans le cas d'une structure par exemple une barre sous flexion simple la situation est quasi identique. En effet une fois la fissure amorcée, l'amplitude du facteur d'intensité des contraintes est une fonction croissante de la longueur de fissure; il n'y a donc pas de possibilité d'arrêt de la fissure et la rupture survient rapidement.

En fatigue mécanique oligocyclique l'amorçage sur tous les sites commence quasiment au même moment (la bande de dispersion est moins large qu'en fatigue à grand nombre de cycles). Par ailleurs une grand partie de la durée de vie est en propagation. Une fois une fissure amorcée sur un site la durée (par rapport à l'amorçage) de la propagation jusqu'à rupture est suffisamment longue pour permettre l'amorçage sur d'autres sites. Il peut donc exister un réseau dense.

En fatigue thermomécanique, des essais sur éprouvettes montrent [4] que pour des durées de vie de dix à cent mille cycles il n'y a pas de différence entre la fatigue thermomécanique et la fatigue mécanique au niveau du comportement du matériau. On ne possède pas de résultats pour des nombres de cycles plus élevés (essais trop longs). Pour une structure soumise à un chargement thermique périodique, il est possible de montrer qu'il y a une possibilité d'arrêt des fissures dans la profondeur à cause d'un gradient spatial de contrainte très important dû aux hautes fréquences du chargement thermique. La figure 1 montre les variations des facteurs d'intensité des contraintes en mode I sous l'hypothèse de l'élasticité pour une plaque contenant une fissure bande soumise à un chargement sinus. Comme on peut le constater,
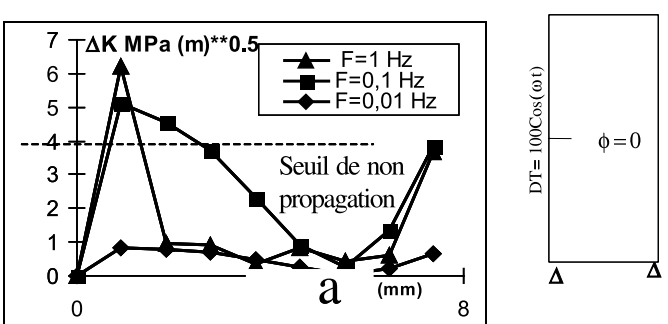

Fig. 1. Facteurs d'intensité des contraintes en fonction de la fréquence du chargement thermique.

pour certaines fréquences du chargement thermique l'amplitude du facteur d'intensité des contraintes après avoir augmenté, chute de façon importante dans la profondeur et donc peut passer en dessous du seuil de non propagation. Ainsi une première fissure amorcée sur un site de concentration de contrainte s'arrête dans la profondeur. Une deuxième a alors la possibilité (le temps) de s'amorcer si elle est en dehors de la zone d'écrantage de la première fissure. Si cette deuxième fissure est à son tour arrêtée une troisième peut s'amorcer et ainsi de suite jusqu'à ce qu'un réseau dense de fissures se forme.

Pour le cas d'une fissure semi-elliptique (extrémités $A$ et $B$ ) dans un parallélépipède soumis à un chargement de flexion pure, la figure 2 montre les facteurs d'intensité des contraintes aux points A et C. Ces calculs sont réalisés pour une valeur de $a / t$ égale à $0,2[5]$ où $t$ représente l'épaisseur de la plaque et a la profondeur maximale de la fissure. La présence d'un réseau dense à la surface s'explique de la façon suivante : pour $K_{\mathrm{I}}(\mathrm{A})>K_{\mathrm{I}}(\mathrm{C})$ (resp. $\left.K_{\mathrm{I}}(\mathrm{A})<K_{\mathrm{I}}(\mathrm{C})\right)$ c'est en A (resp. C) que la propagation en fatigue est plus rapide. La propagation s'arrête quand $K_{\mathrm{I}}(\mathrm{A})=K_{\mathrm{I}}(\mathrm{C})=K_{\text {th }}$ (où $K_{\text {th }}$ est le seuil de non propagation). On peut conclure qu'une fois le point A (en profondeur) bloqué le point $\mathrm{C}$ le sera aussi plus ou moins tardivement. L'analyse présentée ici est confirmée à notre point de vue par les essais du CEA en fatigue thermique [6] même si cette analyse n'est pas présentée dans [6]. Pour le cas de la flexion la position d'équilibre 


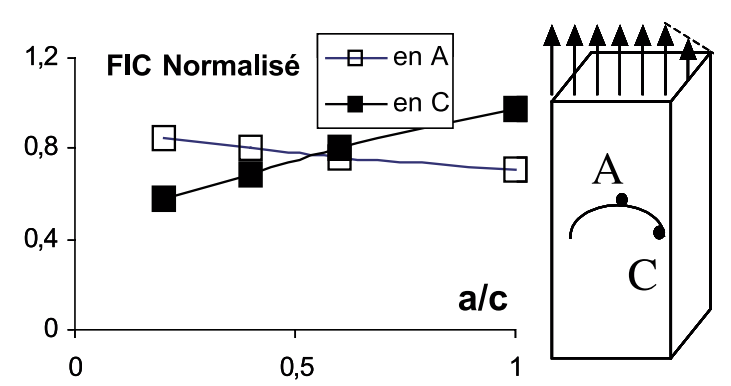

Fig. 2. Les FICs dans la profondeur et sur la surface pour différents rapports d'excentricité $a / c$.

correspond à une demi-ellipse d'excentricité $a / c=0,6$. Pour le cas de la traction la position d'équilibre est quasiment un demi cercle. On peut raisonnablement penser que pour des gradients importants issus du chargement thermique (contrainte très décroissante dans l'épaisseur) les fissures sont encore plus allongées (rapport $a / c$ plus petit que 0,6). Les résultats d'expertise sur les composants montrent que pour les épaisseurs de tuyauterie de l'ordre de 9,2 $\mathrm{mm}$ la profondeur d'arrêt est de 2,5 $\mathrm{mm}$ et pour les épaisseurs de $12,5 \mathrm{~mm}$ cette profondeur est de $2 \mathrm{~mm}$. Par ailleurs on montre que le rapport $a / c$ peut atteindre la valeur de 0,05 [7].

\subsection{La multi-directionnalité d'un réseau de faïençage thermique}

Pour un long tube soumis à une température constante sur la peau interne les valeurs des contraintes axiale et circonférentielle sont quasiment identiques. Néanmoins à cause des facteurs aggravants (contrainte moyenne, rayures de tournage) l'amorçage n'est pas simultané dans les deux directions. Pour des hautes fréquences d'un chargement thermique les fissures peuvent s'arrêter à une certaine profondeur. Ainsi quand les fissures amorcées dans une direction s'arrêtent, l'amorçage et la propagation ont le temps de se produire dans une deuxième direction. On peut donc conclure que deux catégories de fissures, orthogonales entre elles, peuvent coexister et qu'avec le temps des réseaux uniaxiaux deviennent biaxiaux en absence de rupture.

\section{L'effet du grenaillage en fatigue thermique à grand nombre de cycles}

L'opération du grenaillage est généralement utilisée pour améliorer la durée de vie en fatigue, parce qu'elle crée une contrainte de compression à la surface du métal. Cette opération crée également un écrouissage important. Pour les aciers inoxydables sous déformation cyclique contrôlée, contrairement au cas de la contrainte contrôlée, cet écrouissage est pénalisant et peut annuler l'effet bénéfique de la contrainte de compression.

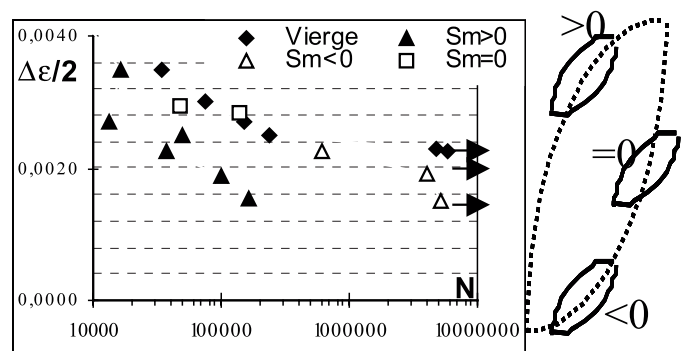

(a)

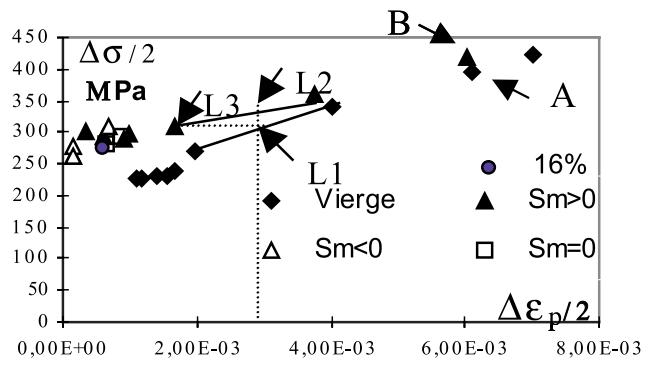

(b)

Fig. 3. Courbes de fatigue et d'écrouissage cyclique avec contraintes moyennes $=0,>0,<0$.

\subsection{Effet du pré-écrouissage en déformation et en contrainte contrôlée}

Des essais expérimentaux réalisés sur des éprouvettes cylindriques en acier austénitique $304 \mathrm{~L}$ et $316 \mathrm{~L}$ ont montré [3] qu'un pré-écrouissage cyclique peut améliorer la durée de vie en contrainte imposée alternée et la réduire en déformation imposée alternée (Fig. 3a). Par contre on montre que pour un acier ferritique A42 l'effet du pré-écrouissage est négligeable. Dans ces essais, le pré-écrouissage cyclique est de $+/-2 \%$ pour 10 cycles. Pour différencier l'effet du pré-écrouissage et l'effet de la contrainte moyenne des essais ont été réalisés pour des contraintes moyennes, positive, négative, et nulle. On peut noter que même sous une contrainte de compression (-100 MPa [3]) la durée de vie après le préécrouissage reste inférieure à la durée de vie sans préécrouissage. Par ailleurs un pré-écrouissage monotone important $16 \%$ réduit également la durée de vie en déformation contrôlée [3]. L'effet bénéfique d'un sablage en contrainte contrôlée a été montré [7] au travers de l'effet du pré-écrouissage en opposition avec la présence des réseaux de faïençage sur certains composants dans une zone de compression de $-500 \mathrm{MPa}$ en fatigue thermique. On peut conclure finalement que l'opération du grenaillage est susceptible d'avoir un effet pénalisant en déformation contrôlée, néanmoins elle reste toujours bénéfique en contrainte contrôlée.

L'explication repose [8] sur la dépendance de la courbe cyclique en pré-écrouissage. La figure $3 \mathrm{~b}$ montre pour un acier $304 \mathrm{~L}$, à la température ambiante, des courbes d'écrouissage cyclique pour un cas uniaxial de tractioncompression. La courbe A montre une courbe cyclique obtenue sur des éprouvettes vierges. La courbe B montre 
la courbe cyclique obtenue après un pré-écrouissage cyclique de $+/-2 \%$. Des résultats semblables existent sur l'acier 316 qu'on explique par le phénomène de surécrouissage. Par contre sur un acier ferritique A42 on ne détecte pas ce phénomène. Nous allons supposer qu'entre deux états stabilisés cycliquement ayant la même amplitude de déformation (resp. amplitude de contrainte) celui ayant l'amplitude de contrainte (resp. amplitude de déformation) la plus grande est le plus endommageant. $\mathrm{Si}$ maintenant on compare les deux points L1 et L2, on observe qu'ils ont la même amplitude de déformation mais on note que l'amplitude de contrainte est plus grande après pré-écrouissage qu'avant. Ainsi l'endommagement cyclique est plus grand en L2 qu'en L1. Un pré-écrouissage (ici cyclique) a donc un effet pénalisant sur la durée de vie en déformation contrôlée pour un acier 304L. Par contre si on compare les points L1 et L3, on a la même amplitude de contrainte mais l'amplitude de déformation est plus petite en L3 qu'en L1. Ainsi l'endommagement cyclique est plus petit en L3 qu'en L1. Un pré-écrouissage a donc un effet bénéfique sur la durée de vie en contrainte contrôlée pour un acier 304L. On montre un résultat analogue pour un pré-écrouissage monotone important.

\subsection{Simulation de l'effet du pré-écrouissage avec un modèle polycristallin}

Nous avons tenté de valider l'analyse précédente par une simulation avec le modèle de comportement polycristallin de l'ENSMP [9] qui est mis au point pour décrire le phénomène de sur-écrouissage. Les figures 4a et $4 \mathrm{~b}$ montrent l'effet du pré-écrouissage cyclique sur les cycles limites pour un acier 316L (calculs réalisés par le Code_Aster). On remarque que sous contrainte contrôlée (Fig. 4a) après pré-écrouissage le comportement devient élastique, donc beaucoup moins endommagent qu'avant pré-écrouissage. Par contre en déformation contrôlée (Fig. 4b) après pré-écrouissage il faut une amplitude de contrainte plus importante pour créer la même amplitude de déformation qu'avant le pré-écrouissage

\section{Soudage et l'effet pénalisant de la contrainte moyenne de traction}

Des résultats de mesures de contraintes résiduelles de soudage montrent l'apparition de réseaux de faïençage dans les zones où la contrainte résiduelle de traction est maximale. La figure 5a montre la configuration d'un réseau de faïençage sur un composant. La figure $5 \mathrm{~b}$ montre le résultat de simulation des contraintes résiduelles axiale et circonférentielle sur la peau interne d'un tube mince chanfreiné [2] d'une épaisseur environ égale à celle d'un circuit de refroidissement à l'arrêt (Fig. 5a). Sur la figure 5b on constate :

- un effet local [2] de traction aux abords de la soudure sur une distance d'environ l'épaisseur du tube, avec une contrainte circonférentielle nettement plus élevée

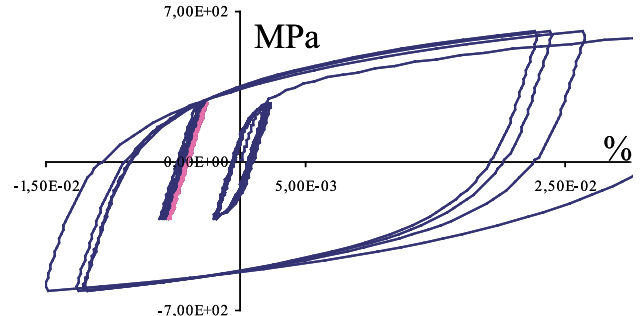

(a)

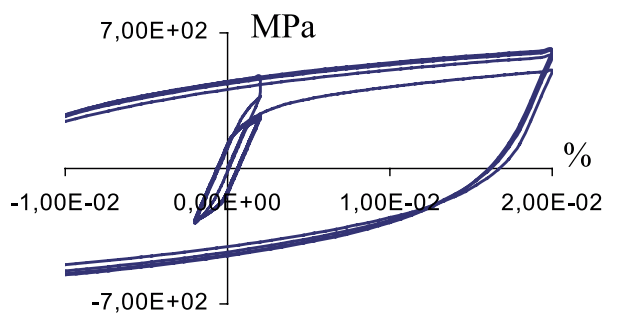

(b)

Fig. 4. (a) Modèle polycristallin, effet du pré-écrouissage en contrainte contrôlée. (b) Modèle polycristallin, effet du préécrouissage en déformation contrôlée.

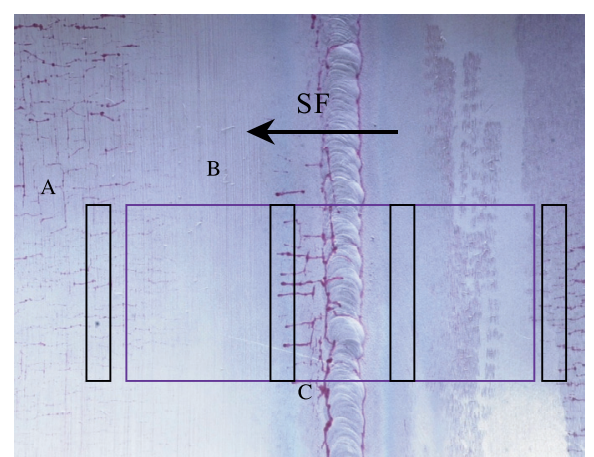

(a)

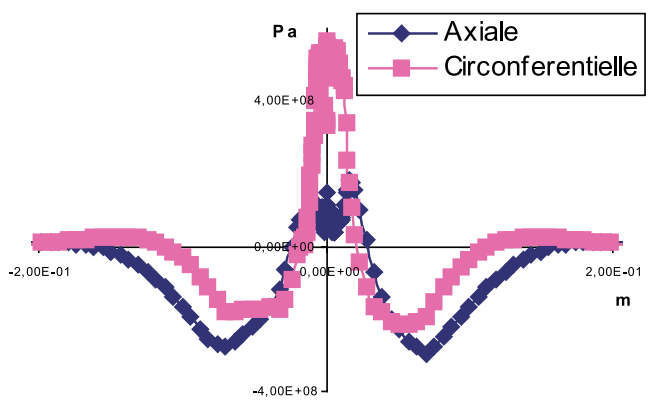

(b)

Fig. 5. Explication des réseaux de faïençage par la présence des contraintes résiduelles.

que la contrainte axiale. Cet effet local (contrainte circonférentielle) semble expliquer au travers de l'effet pénalisant d'une contrainte moyenne de traction la présence des fissures orthogonales à la soudure ;

- un effet global [2] (solution coque) de compression sur une distance de l'ordre de $\sqrt{R_{\mathrm{m}} * h}$ pour les deux composants de contrainte. Cet effet global 


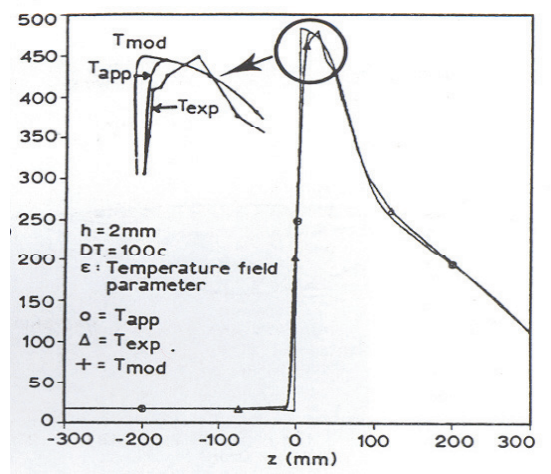

(a)

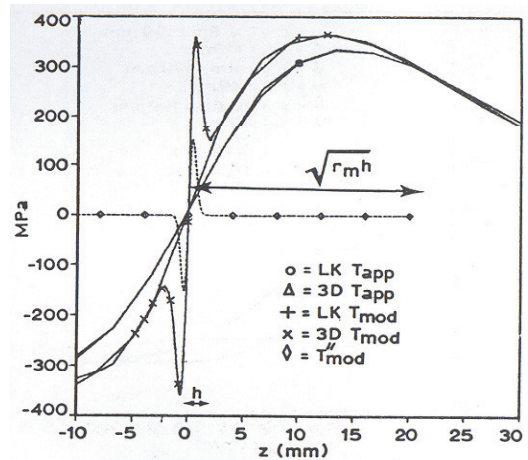

(b)

Fig. 6. Effet local et sa dépendance en la dérivée seconde du chargement thermique.

semble expliquer au travers de l'effet bénéfique de la contrainte moyenne en compression les zones saines mitoyennes à la soudure.

Ces résultats sont néanmoins en contradiction avec des résultats de tests uniaxiaux en contrainte contrôlée sur un acier 304L, qui montrent un effet bénéfique de la contrainte moyenne de traction sur la durée de vie pour une éprouvette lisse [7]. Par ailleurs les résultats de [7] sont en opposition avec un effet net et pénalisant de la contrainte moyenne de traction en déformation contrôlée (Fig. 3). L'ensemble de ces résultats confirme la différentiation entre des chargements en contrainte et en déformation contrôlée. Nous expliquons les résultats de la référence [7] par une amélioration de la durée de vie en amorçage suite à l'augmentation de la contrainte maximale dans un essai avec contrainte moyenne comparé à un essai avec contrainte moyenne nulle, induisant ainsi un pré-écrouissage plus important.

\subsection{Effet de la dérivée seconde de température}

La figure 6a montre un choc thermique ( $\left.T_{\text {exp }}\right)$ axial subi par un tube mince. La température $T_{\exp }$ a été approchée par deux champs de température pratiquement superposables, $T_{\text {mod }}$ et $T_{\text {app }}$, mais avec des dérivées secondes différentes [10]. Dans la figure $6 \mathrm{~b}$ on montre que les solutions globales sont proches pour les deux approximations de la température expérimentale. Par contre, les solutions locales sont très différentes (nulle pour $T_{\text {app }}$ ). Ceci montre l'importance de la dérivée seconde de la température sur la solution locale. Des résultats analogues sont obtenus en élastoplasticité. Une difficulté essentielle pour la simulation des contraintes résiduelles est l'évaluation du champ de température de soudage, ainsi il peut exister une marge d'erreur importante sur l'évaluation de l'effet local. Ceci montre que l'explication du faïençage des circuits de refroidissement à l'arrêt reste pour l'instant très qualitative.

\section{Références}

[1] S. Taheri, An attempt to analyse crack initiation under high cycle thermal fatigue on a mixing zone of auxiliary cooling System, in : Proceeding of 7 th conference on biaxial \& multiaxial fatigue, Berlin, 2004, pp. $549-555$

[2] S. Taheri, High cycle thermal fatigue in a mixing zone of auxiliary cooling system, in : Proceeding of ASME-PVP Conference, San Diego, 2004

[3] V. Doquet, S. Taheri, Effet d'un pré-écrouissage ou d'un sur-écrouissage sur la durée de vie en fatigue de divers aciers à contrainte ou déformation imposée, Revue Française de Mécanique 1 (2000) 27-33

[4] N. Hadar, Fatigue thermique d'un acier inoxydable austénitique 304L : simulation de l'amorçage et de la croissance des fissures courtes en fatigue isotherme et anisotherme, Thèse de doctorat, École des mines de Paris, 2003

[5] I.S. Rajou, S.N. Atluri, J.C. Newman, Stress intensity factors for small surface and corner cracks in plates, in : Wei/Gangolf, editors, Fracture mechanics perspectives \& directions ASME STP 1020 (1999) 297-316

[6] V. Maillot, A. Fissolo, G., Degalaix, S. Degalaix, Thermal fatigue crack networks parameters stability and experimental study, Int. J. of solides and structures 42(1) (2005) 759-764

[7] F. Cornuel, F. Fradet, Rapport EDF/DPN D5710/IECH/2002/008081/1, 2004

[8] S. Petitjean, Influence de l'état de surface sur le comportement en fatigue à grand nombre de cycles de l'acier inoxydable austénitique 304L, Thèse, Université de Poitiers, 2003

[9] S. Taheri, Low cycle fatigue damage cumulation rule for non proportional loading, in : A. Pineau, G. Cailletaud, T.C. Lindley (ed.), Mechanical Engineering Publications (Multiaxial and fatigue design), London, 1996, pp. 283299

[10] G. Cailletaud, A micromechanical approach to inelastic behaviour of metals, Int. J. Plasticity 8 (1992) 55-73

[11] S. Taheri, Threedimensional local effect and shell theory. Int J. Pres Ves \& Piping 36 (1989) 225-246 